\title{
Insertion of Transnasal Abscess Drainage Tube, Decompression Tube, and Jejunal Nutrition Tube Under Fluoroscopy to Treat Esophagojejunal Anastomotic Fistula After Gastrectomy for Gastric Cancer
}

\section{Yue Zhao}

Department of Interventional Radiology, The First Affiliated Hospital of Zhengzhou University Meipan Yin

Department of Interventional Radiology, The First Affiliated Hospital of Zhengzhou University Tao Liu

Department of Gastrointestinal Surgery, The First Affiliated Hospital of Zhengzhou University

\section{Zhen Yang}

Department of Gastrointestinal Surgery, The First Affiliated Hospital of Zhengzhou University

\section{Yaozhen Ma}

Department of Interventional Radiology, The First Affiliated Hospital of Zhengzhou University Chunxia Li

Department of Interventional Radiology, The First Affiliated Hospital of Zhengzhou University Xiaobing Li

Department of Interventional Radiology, The First Affiliated Hospital of Zhengzhou University Shuai Wang

Department of Interventional Radiology, The First Affiliated Hospital of Zhengzhou University Pengfei Xie

Department of Interventional Radiology, The First Affiliated Hospital of Zhengzhou University

Gang Wu ( $\nabla$ wuganghenan2004@zzu.edu.cn )

Department of Interventional Radiology, The First Affiliated Hospital of Zhengzhou University

\section{Research Article}

Keywords: Gastrectomy, anastomotic fistula, complication, interventional radiology

Posted Date: February 3rd, 2021

DOI: https://doi.org/10.21203/rs.3.rs-149618/v1 
License: (c) (i) This work is licensed under a Creative Commons Attribution 4.0 International License. Read Full License 


\section{Abstract \\ Background}

Esophagojejunal anastomotic leakage is a serious complication after total gastrectomy. This study evaluated the safety and efficacy of transnasal placement of drainage tube, jejunal decompression tube, and jejunal nutrition tube under fluoroscopy for treatment of esophagojejunal anastomotic fistula after gastrectomy in gastric cancer patients.

\section{Methods}

Retrospective review of patients with esophagojejunal anastomotic fistula treated with transnasal placement of abscess drainage tube, decompression tube, and jejunal nutrition tube under fluoroscopy. Fistula healing time, patient survival, and Eastern Cooperative Oncology Group (ECOG) performance status before and after treatment were evaluated.

\section{Results}

Thirty-eight patients were included in the study. Insertion of the transnasal abscess drainage tube, jejunal decompression tube, and nutrition tube was successful on the first attempt in all patients. Simple transnasal drainage was used in 27 patients (one drainage tube placed plus one drainage tube replacement in 15 patients, and one drainage tube placed in 12 patients), and transnasal drainage plus percutaneous abscess drainage in 11 patients. After placement of the tube, the mean volume of drainage was $120 \mathrm{ml}(10-850 \mathrm{~mL})$; the amount steadily decreased from then on. The fistula healed in 33 patients. Median time to fistula healing was 78 days (6-248 days). Two patients suffered gastrointestinal bleeding after the procedure but one patient died.

\section{Conclusions}

Transnasal insertion of transnasal abscess drainage tube, jejunal decompression tube, and jejunal nutrition tube under fluoroscopy appears to be a simple, minimally invasive, effective, and safe method for treating esophagojejunal anastomotic fistula after gastrectomy for gastric cancer.

\section{Background}

Esophagojejunal anastomotic fistula develops in $2-15 \%$ of patients receiving total gastrectomy and esophagojejunostomy for treatment of gastric cancer and can seriously impact quality of life and prognosis; the mortality rate exceeds $50 \%$ [1-3]. Early clinical manifestations of the fistula are an increase in the amount of drainage, pus-like changes in the drainage fluid, or poor drainage and intermittent fever. Without intervention, abscesses may form and lead to persistent high fever and 
abdominal pain, and complications such as peritonitis, mediastinitis, chest infection, multiple organ failure, sepsis, respiratory failure, and death. The presence of esophagojejunal fistula can be confirmed by the methylene blue test, esophagography, or endoscopy [3-4].

Currently, there is no consensus on the best treatment for esophagojejunal fistula. Treatments include fasting and nil water through mouth with complete parenteral nutritional support, mucosal protective agents, gastrointestinal decompression, percutaneous thoracic drainage tube placement, surgery-related drainage tube placement, surgical repair, esophageal stents, titanium clips, and endoluminal vacuum treatment (EVT) $[1,3,5-6]$.

Interventional treatment of mediastinal abscess after spontaneous esophageal rupture and esophageal cancer has been shown to be safe and effective [7-8]. However, there are no reports in literature of interventional treatment of esophagojejunal anastomotic fistula. At our hospital we have been treating esophagojejunal anastomotic fistula by transnasal placement of abscess drainage tube, jejunal decompression tube, and jejunal nutrition tube under fluoroscopy. The aim of this study is to report our 8year experience with the use of this interventional procedure.

\section{Materials And Methods}

The data of patients with esophagojejunal fistula treated by interventional therapy at our hospital between June 2012 and May 2020 were retrospectively reviewed. Patients were eligible for inclusion in this study if they 1) had undergone total gastrectomy and esophagojejunostomy for treatment of gastric cancer; 2) had esophagojejunal fistula confirmed by the methylene blue test, imaging, or endoscopy; and 3) had received transnasal placement of abscess drainage tube, jejunal nutrition tube, and jejunal decompression tube under fluoroscopy. Patients were excluded if 1 ) they had esophagogastric anastomotic fistula after non-total gastrectomy; 2) they had duodenal stump fistula; or 3) the esophagojejunal fistula had healed after conservative treatment, surgical treatment, or endoscopic treatment.

The following data were collected for analysis: sex, age, and comorbidities; history of gastric cancerrelated chemotherapy; pre-procedure American Society of Anesthesiologists (ASA) score and Procedural Severity Score (PSS) [9]; pre- and post-procedure laboratory test results and Eastern Cooperative Oncology Group (ECOG) performance status; time from surgical resection to diagnosis of fistula; fistula size, location, and classification (according to Clavien-Dindo classification [10]); time from diagnosis of fistula to interventional treatment; abscess cavity extent and presence of communications; volume of drainage from abscess cavity; pus culture results during treatment and follow-up; morphological changes in fistula and abscess cavity; complications (infection, bleeding, shock, and so on); laboratory and imaging examination results; ECOG performance status at last follow-up; time of healing of fistula or death; and cause of death.

Ethics committee of our hospital approved this study; the need for informed consent was waived because of the retrospective nature of the study. 


\section{Preoperative preparation}

Preoperatively, all patients underwent blood routine examination, liver and kidney function tests, serum electrolytes estimation, electrocardiogram, methylene blue test, upper gastrointestinal contrast study, and plain and enhanced chest CT scan. Pre-procedure preparation was with fasting, gastric mucosal protective agents, parenteral nutrition, and prophylactic antibiotics. Patients with dyspnea or oxygen saturation $<90 \%$ received oxygen by nasal cannula and, if necessary, tracheal intubation and ventilatorassisted breathing.

\section{Procedure}

Esophagography was performed first. The nasal cavity, pharynx, and esophagus were anesthetized with tetracaine gel 10 min after the esophagography. With the patient supine on the digital subtraction angiography table, ioversol was administered orally. Frontal and $45^{\circ}$ left anterior oblique views of the esophagus were studied to determine the position and size of the esophagojejunal anastomotic leakage and the extent of spillage of contrast.

\section{Transnasal insertion of jejunal nutrition tube and decompression tube}

Under fluoroscopy, a 0.035-inch hydrophilic membrane guide wire (Cook Medical LLC, Bloomington, IN, USA) and a 5F vertebral artery catheter (Cook Medical) were passed through one nostril and advanced through the pharynx and esophagus, across the esophagojejunal anastomosis area into the upper jejunum. The catheter was withdrawn, and a No. 14 jejunal nutrition tube was passed over the guide wire 40-50 cm into the jejunum. In the same way, a decompression tube was inserted through the other nostril into the jejunum 5-10 $\mathrm{cm}$ beyond the esophagojejunostomy (Fig.1).

\section{Transnasal insertion of abscess drainage tube}

The drainage catheter and guide wire were introduced along the side of the jejunal decompression tube into the esophagus. Transcatheter esophagography was performed to display the location of the esophagojejunal fistula, and the catheter and guide wire were then advanced into the abscess cavity through the fistula. The lowest pole of the abscess cavity was gently probed, and a 5F PERFORMA® vessel catheter (Merit Medical, USA) was exchanged and advanced until the tip was at the lowest pole of the abscess cavity. If the abscess cavity was large, a $5 \mathrm{~F}$ pigtail catheter (Merit Medical) was inserted into the abscess cavity in the same way (Fig.1).

\section{Percutaneous abdominal drainage tube replacement}

For patients with a percutaneous abscess drainage tube inserted during previous surgery, the tube was exchanged for a $10.2 \mathrm{~F}$ or $12 \mathrm{~F}$ external drainage tube, which was then connected to a negative pressure suction system up to $-125 \mathrm{mmHg}$.

\section{Postoperative treatment and follow-up}


Post procedure, the patients received fasting, jejunal nutrition, appropriate intravenous nutritional supplements, and continuous negative-pressure suction through the abscess drainage tube. Antibiotics were prescribed according to culture results. Normal-saline lavage was performed 1-2 times per day through the transnasal or percutaneous drainage tube.

Esophagography and chest CT were repeated 5-7 days after the procedure to assess abscess cavity size and the efficacy of suction. When the distal part of the abscess cavity had healed, the position of the drainage tube was adjusted under fluoroscopy so that the tip was at the proximal part (Fig.2). The position of the drainage tube was adjusted every 2 weeks after checking the healing of the abscess cavity; when necessary, the drainage tube was replaced. The percutaneous drainage tube was removed when 5-7 days had passed without any drainage. The transnasal abscess drainage tube was removed when contrast injected into the cavity showed only a thin line. The jejunal feeding tube and decompression tube were retained for another week. Oral intake was restarted after esophagography confirmed healing of the fistula.

\section{Outcomes}

Follow-up quality of life was evaluated by ECOG performance status. The effectiveness of treatment was defined as the reduction of the abscess cavity by $>50 \%$ in an individual patient under radiography of the abscess and the improvement of the patient's ECOG performance status, which was recorded at the first admission and the last follow-up. Treatment success was defined as complete disappearance of the abscess and healing of the fistula, with no recurrence over 6 months of follow-up. Treatment failure was defined as persistence of abscess cavity or fistula, or fistula recurrence within 6 months. Need for surgical intervention, or death due to esophagojejunal leak-related complications during treatment, was also regarded as treatment failure.

\section{Results}

A total of 38 patients (31 males, 7 females; mean age, $61.8 \pm 9.6$ years) were included in this study (Table 1). R0 resection was achieved in all patients. Postoperative pathology showed lymph node metastasis in 18 patients. Among the 38 patients, 6 received chemotherapy before the surgery and 1 received chemotherapy in the interval between the surgery and the interventional procedure; 12 patients received intraperitoneal chemohyperthermia with fluorouracil during surgery. Four of the patients had been diagnosed with fistula at other hospitals and had been transferred to our center after failure of endoscopic treatment ( 1 patient with esophageal stent, 1 patient with titanium clip, and 2 patients with gastric tube). All four patients were offered the interventional therapy only after further surgical or endoscopic treatments were ruled out by the concerned specialists. 
Table 1

Characteristics of the study population $(n=38)$

\begin{tabular}{|c|c|}
\hline Characteristic & $\mathbf{n}$ \\
\hline \multicolumn{2}{|l|}{ Sex } \\
\hline Male & 31 (mean age, $61.3 \pm 8.6$ years) \\
\hline Female & 7 (mean age, $66.4 \pm 12.5$ years) \\
\hline \multicolumn{2}{|l|}{ Comorbidity } \\
\hline Hypertension & 7 \\
\hline Cancer cachexia & 7 \\
\hline Diabetes & 5 \\
\hline \multicolumn{2}{|l|}{ Clinical stage } \\
\hline Stage I & 5 \\
\hline Stage II & 16 \\
\hline Stage III & 15 \\
\hline Stage IV & 2 \\
\hline \multicolumn{2}{|l|}{ Pathological type } \\
\hline Adenocarcinoma & 36 \\
\hline Squamous cell carcinoma & 1 \\
\hline Neuroendocrine carcinoma & 1 \\
\hline \multicolumn{2}{|l|}{ Differentiation } \\
\hline Poorly differentiated & 13 \\
\hline Moderately differentiation & 24 \\
\hline Well differentiated & 1 \\
\hline \multicolumn{2}{|l|}{ Method of fistula diagnosis } \\
\hline Esophagography & 21 \\
\hline Methylene blue test & 10 \\
\hline CT & 6 \\
\hline Resurgery for suspected intraperitoneal bleeding & 1 \\
\hline
\end{tabular}

The most common symptom before the interventional procedure was fever (32/38), and the most common signs were increased drainage and or pus-like changes in the discharge (32/38). The mean 
white blood cell count before the interventional procedure was $11.53 \pm 4.60 \times 10^{12}$. The median time from surgery to diagnosis of fistula was 7.5 days (1-36 days). By the Clavien-Dindo classification, 10 fistulas were type III and 28 were type IV. Mean pre-procedure ASA score was $3.58 \pm 0.64$, mean PSS was $8.50 \pm$ 2.77, and mean ECOG score was $3.11 \pm 0.75$. The mean time from fistula diagnosis to the interventional procedure was 31.84 days (4-344 days) in all patients.

Transnasal insertion of abscess drainage tube, jejunal decompression tube, and jejunal nutrition tube was successful on the first attempt in all patients. The average fistula diameter was $6.16 \mathrm{~mm}$ (range, 1.8-11.9 mm). While 27 patients (one drainage tube placed plus one drainage tube replacement in 15 patients, and one drainage tube placed in 12 patients) received abscess drainage only through the transnasal route, 11 patients received abscess drainage through transnasal plus percutaneous route.

The mean volume of drainage was $120 \mathrm{~mL}(10-850 \mathrm{~mL})$ on day 1 ; the amount decreased steadily from then on. The mean white blood cell count decreased to $8.25 \pm 3.83 \times 10^{12}$ on day 3 after the intervention procedure. Culture of drainage fluid showed no growth in 11 patients; a single organism was grown in 12 patients and two or more organisms in 15 patients. The most commonly grown organisms were Escherichia coli (11/38), Klebsiella pneumoniae(9/38), and Pseudomonas aeruginosa (8/38). During treatment, there were 27 episodes of blockage of the abscess drainage tube ( $0-3$ times/patient) and 18 episodes of breakage of the abscess drainage tube ( $0-3$ times/patient); displacement, requiring readjustment of the abscess drainage tube position, occurred a mean 3.2 times per patient (range $0-11$ times).

\section{Follow-up}

Follow-up data were available until December 2020. Median follow-up was for 16 months (range, 1 month to 8 years). Common complications were weight loss (19/38) and reflux (22/38). The fistula healed in 33 patients, but treatment success was completed in only 32 patients; 1 patient died of septic shock 2 months after fistula disappearance. The median healing time was 78 days ( $6-248$ days). The mean ECOG score of fistula healing patients was $2.14 \pm 0.21$. Among the 33 patients, 17 patients survived (survival time, 6 months -8 years) and 16 patients died (survival time, 2 months -5 years). The most common cause of death in patients with fistula healing was tumor recurrence $(11 / 33)$.

The fistula did not heal in 5 patients; all died. In this cohort, the median survival time was 75 days (2 days-8 months) and the median time from diagnosis to interventional treatment was 27.5 days (7-34 days). Causes of death included septic shock and malignant consumption (Table 2). 
Table 2

Outcomes

\begin{tabular}{|lll|}
\hline & Patients with healed fistula & Patients with unhealed fistula \\
\hline Final outcome & 17 & 0 \\
Survived & 16 & 5 \\
\hline Died & 11 & 0 \\
Cause of death & 1 & 4 \\
Tumor recurrence & 4 & 0 \\
Septic shock & 0 & 1 \\
Cachexia & & \\
Gastrointestinal bleeding & & \\
\hline
\end{tabular}

\section{Complications}

During interventional procedure, there were no cases of hemorrhage, suffocation, or cardiorespiratory arrest. One patient had gastrointestinal hemorrhage 2 days after the interventional procedure, but it was controlled by emergency upper gastroduodenal artery embolization. The mortality rate was $2.6 \%(1 / 38)$ within 30 days of the interventional procedure, that was due to gastrointestinal bleeding.

\section{Discussion}

Onset of esophagojejunal anastomotic leakage, which generally occurs 5-12 days after surgery, predicts poor outcome; even with treatment, the mortality rate is in the range of $18-65 \%$ [3]. There is still no consensus on the best treatment for esophagojejunal fistula; however, the key principles of treatment are isolation of the fistula, adequate drainage, nutritional support, and antibiotic therapy [11].

In this study, transnasal placement of abscess drainage tube, jejunal decompression tube, and jejunal nutrition tube was performed under local anesthesia in awake patients. The success rate was $100 \%$ on the first attempt. We found that the symptoms begin to improve immediately after abscess drainage tube insertion.

Currently used treatments include endoscopic titanium clip, over-the-scope clip (OTSC), esophageal stent, and EVT. Although these methods are effective, they have limitations. Esophageal stent is associated with many complications, the most common being displacement. Even with use of titanium clips or other measures to fix the stent, displacement occurs in $75-90 \%$ of cases [13-15]. Titanium clips, OTSC, and EVT have been successfully used to treat large fistulas $(>2 \mathrm{~cm})$ and chronic fistulas [1], but they are technically demanding procedures and may sometimes have to be performed under general anesthesia; 
therefore, they cannot be used in critically ill patients with poor cardiopulmonary function. According to literature on treatment of esophageal fistula, it is necessary to change the sponge after 3-7 days in EVT [16-17]; moreover, the procedure has to be performed under gastroscopy, which is expensive and cumbersome. Secondary surgical repair is a supplementary method for treatment of esophagojejunal anastomotic leakage. It is indicated in patients who have failed endoscopic treatment or in those with severe complications such as intra-abdominal hemorrhage, sepsis, and giant anastomotic leakage; however, surgical repair is unsuitable for patients who have had recent surgery and have hypoproteinemia, or for those with sepsis and unstable blood pressure.

Our method of paranasal insertion of abscess drainage tube, jejunal decompression tube, and jejunal nutrition tube for treatment of esophageal jejunostomy fistula has several advantages. First, it can be performed under local anesthesia in the awake state and so is suitable for critically ill patients with poor cardiopulmonary function. Second, placement of the tubes can be accurately performed under fluoroscopy. Third, the application of continuous suction prevents accumulation of pus and creates a negative pressure environment that is conducive to the healing of the abscess cavity. In theory, it reduces the probability of gastrointestinal bleeding caused by the erosion of arteries in the abscess wall. Fourth, regular esophagography and fistulography through the drainage tube can be performed and the position of the drainage tube adjusted to ensure effective drainage. When necessary, the drainage tube can easily be replaced by the interventional technique under fluoroscopy. Fifth, the procedure cost is low compared with EVT under endoscopy, as EVT requires 3-8 sponge replacements per person per session. Finally, drainage is an established method for treatment of abscesses, and interventional devices are relatively safe. It was reported that the surgical drainage tube was too dumb and too inflexible due to insufficient toughness of the device (24F), and it caused perforation of the digestive tract [18]; in comparison, the catheter guide wire used in our method is very flexible, and its introduction under fluoroscopy is minimally traumatic. So far, there has been no report of bowel perforation resulting from use of interventional devices.

For successful interventional treatment, the intervention should be gentle and precise. Imaging should be performed after placement of the drainage tube to confirm correct positioning of the tube; easy aspiration of contrast agent is proof that the drainage tube is in the right position. Regular imaging of the abscess cavity, with adjustment of the position of the drainage tube when necessary, can accelerate healing. Regular tube care is necessary. We have found that breakage and blockage of the drainage tube are common. This may be because we presently use an angiography catheter as the drainage tube; we hope that a dedicated transnasal abscess drainage tube will become available soon.

The study has limitations. It is a single-center retrospective study and thus, a selection bias is inevitable. Also the sample size is relatively small. Furthermore, there was no control group.

In conclusion, for gastric cancer patients with esophagojejunal fistula after gastrectomy, interventional treatment under fluoroscopy, with transnasal insertion of abscess drainage tube, decompression tube, and jejunal nutrition tube appears to be a feasible, safe, and effective treatment. This simple and 
minimally traumatic procedure can also be performed in very ill patients. Large multicenter controlled studies are needed to confirm our findings.

\section{Declarations}

Ethics approval and consent to participate: The study was approved by the ethics committee of the First Affiliated Hospital of Zhengzhou University. All procedures followed were in accordance with the ethical standards of the responsible committee on human experimentation (institutional and national) and with the Helsinki Declaration of 1975, as revised in 2008. This study is a retrospective analysis, it did not include any human trial, and all participants provided written informed consent.

Consent for publication: Not applicable.

Availability of data and materials: The raw data generated and analyzed in the current study are not publicly available due to protection of patient privacy we can provide data with some basic details related clinical outcomes with shading on their names, etc.. Further information is available from the corresponding author on reasonable request.

Competing interests: All authors declare that they have no competing interests.

Funding: Not applicable.

Authors' contributions $₫$ Authors made substantial contributions in this article. YZ, MPY designed it, searched the literature, collected the raw data, prepared the figures, listed the tables, did the follow up and wrote the original manuscript. TL, ZY provided the gastrectomy surgery. $Y Z M, C X L, X B L, S W$, and $P F X$ operated the interventional procedures and provided pre-and postoperative care. GW put up an original idea and revised the manuscript. All authors read and approved the final manuscript.

Acknowledgements $\square$ We thank all the nurses in both wards and operation room for their best care of all the patients. We appreciate all the editors and anonymous reviewers for their helpful suggestions on improving the quality of our article.

\section{References}

1. Wang W, Qi K, Chang X, Jin Z, Li Z. Two-session endoscopic purse-string suture to close a huge esophagojejunal anastomosis thoracic cavity fistula. Endoscopy. 2019. doi: 10.1055/a-0658-0995.

2. Kawamura H, Ohno Y, Ichikawa N, Yoshida T, Homma S, Takahashi M, et al. Anastomotic complications after laparoscopic total gastrectomy with esophagojejunostomy constructed by circular stapler (OrVil ${ }^{\text {TM }}$ ) versus linear stapler (overlap method). Surg Endosc. 2017. doi: 10.1007/s00464-017-5584-z.

3. Makuuchi R, Irino T, Tanizawa Y, Bando E, Kawamura T, Terashima M. Esophagojejunal anastomotic leakage following gastrectomy for gastric cancer. Surg Today. 2019. doi: 10.1007/s00595-018-1726- 
8.

4. Page RD, Asmat A, McShane J, Russell GN, Pennefather SH. Routine endoscopy to detect anastomotic leakage after esophagectomy. Ann Thorac Surg. 2013. doi:

10.1016/j.athoracsur.2012.09.048.

5. Oka S, Sakuramoto S, Chuman M, Aratani K, Wakata M, Miyawaki Y, et al. Successful treatment of refractory complete separation of an esophagojejunal anastomosis after laparoscopic total gastrectomy: a case report. BMC Res Notes. 2017. doi: 10.1186/s13104-017-2589-6.

6. Jung GM, Lee SH, Myung DS, Lee WS, Joo YE, Jung MR, et al. Novel Endoscopic Stent for Anastomotic Leaks after Total Gastrectomy Using an Anchoring Thread and Fully Covering Thick Membrane: Prevention of Embedding and Migration. J Gastric Cancer. 2018. doi:

10.5230/jgc.2018.18.e2.

7. Wu G, Zhao YS, Fang Y, Qi Y, Li X, Jiao D, et al. Treatment of spontaneous esophageal rupture with transnasal thoracic drainage and temporary esophageal stent and jejunal feeding tube placement. $J$ Trauma Acute Care Surg. 2017. doi: 10.1097/TA.0000000000001272.

8. Wu G, Zeng YW, Wang JX, Ma W, Yin MP, Zhao Y, et al. An interventional radiology technique to treat pharyngeal or esophageal perforation associated with mediastinal abscess in children. J Pediatr Surg. 2020. doi: 10.1016/j.jpedsurg.2019.07.009.

9. Abbas G, Schuchert MJ, Pettiford BL, Pennathur A, Landreneau J, Landreneau J, et al. Contemporaneous management of esophageal perforation. Surgery. 2009. doi: 10.1016/j.surg.2009.06.058.

10. Clavien PA, Sanabria JR, Strasberg SM. Proposed classification of complications of surgery with examples of utility in cholecystectomy. Surgery. 1992.

11. Eisendrath P. Esophageal leaks: extending our toolbox? Endoscopy. 2010. doi: 10.1055/s-00301255687.

12. Rieder E, Dunst CM, Martinec DV, Cassera MA, Swanstrom LL. Endoscopic suture fixation of gastrointestinal stents: proof of biomechanical principles and early clinical experience. Endoscopy. 2012. doi: 10.1055/s-0032-1325730.

13. Persson S, Rouvelas I, Kumagai K, Song H, Lindblad M, Lundell L, et al. Treatment of esophageal anastomotic leakage with self-expanding metal stents: analysis of risk factors for treatment failure. Endosc Int Open. 2016. doi: 10.1055/s-0042-102878. Epub 2016 Mar 30.

14. Kucukay F, Okten RS, Parlak E, Disibeyaz S, Ozogul Y, Bostanci EB, et al. Self-expanding covered metallic stent treatment of esophagojejunal fistulas. Abdom Imaging. 2013. doi: 10.1007/s00261012-9895-1.

15. Oka S, Sakuramoto S, Chuman M, Aratani K, Wakata M, Miyawaki Y, et al. Successful treatment of refractory complete separation of an esophagojejunal anastomosis after laparoscopic total gastrectomy: a case report. BMC Res Notes. 2017. doi: 10.1186/s13104-017-2589-6.

16. Rausa E, Asti E, Aiolfi A, Bianco F, Bonitta G, Bonavina L. Comparison of endoscopic vacuum therapy versus endoscopic stenting for esophageal leaks: systematic review and meta-analysis. Dis 
Esophagus. 2018. doi: 10.1093/dote/doy060.

17. de Moura DTH, de Moura BFBH, Manfredi MA, Hathorn KE, Bazarbashi AN, Ribeiro IB, et al, Thompson CC. Role of endoscopic vacuum therapy in the management of gastrointestinal transmural defects. World J Gastrointest Endosc. 2019. doi: 10.4253/wjge.v11.i5.329.

18. Eleftheriadis E. Drainage-tube penetration into the gastric lumen, mimicking a high-volume enterocutaneous fistula. The significance of postoperative endoscopy. Surg Endosc. 1990. doi: 10.1007/BF02336602.

\section{Figures}




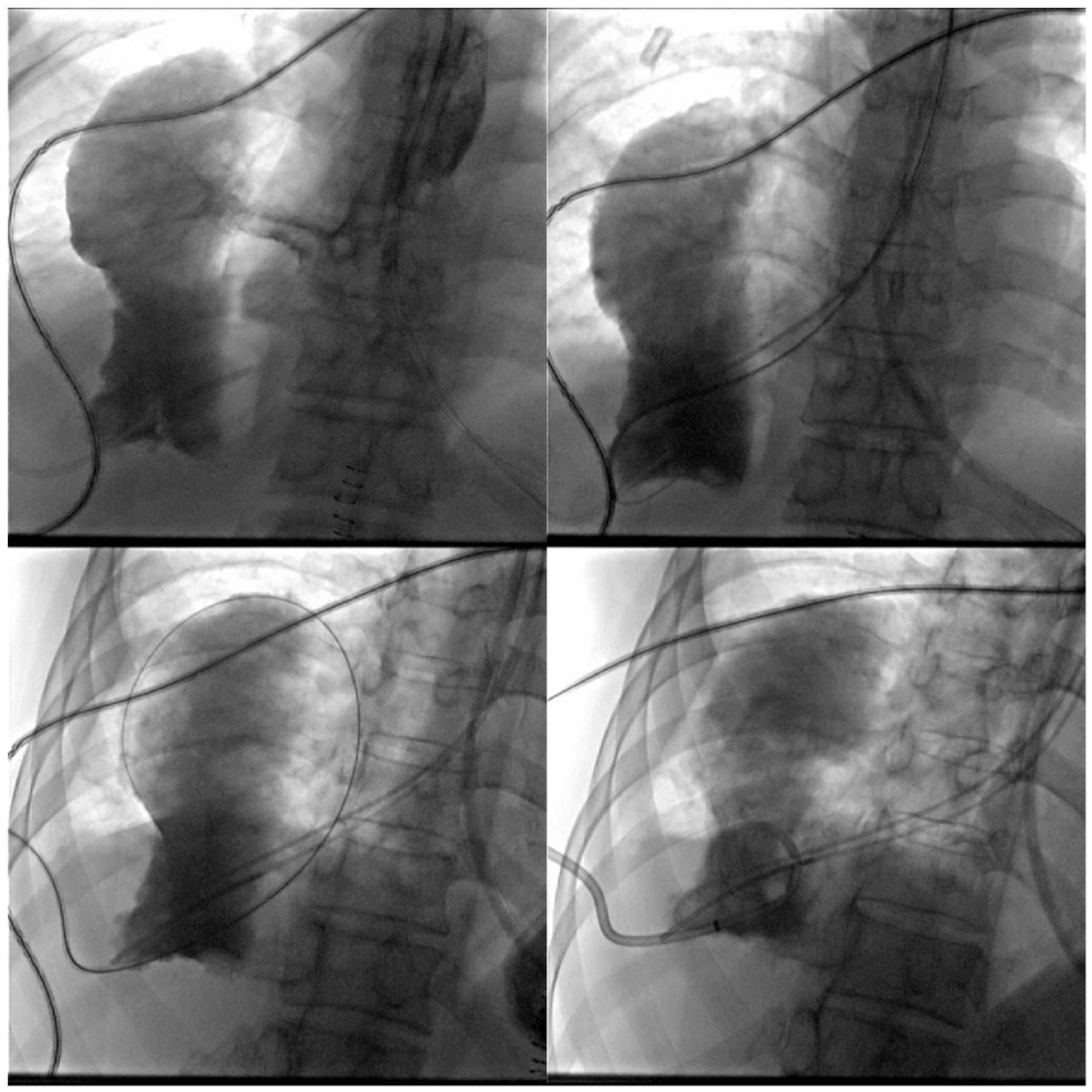

Figure 1

Esophagography shows contrast agent overflow into the right thoracic cavity. An abscess drainage tube was inserted through the nose, and the percutaneous surgical drainage tube was replaced. 


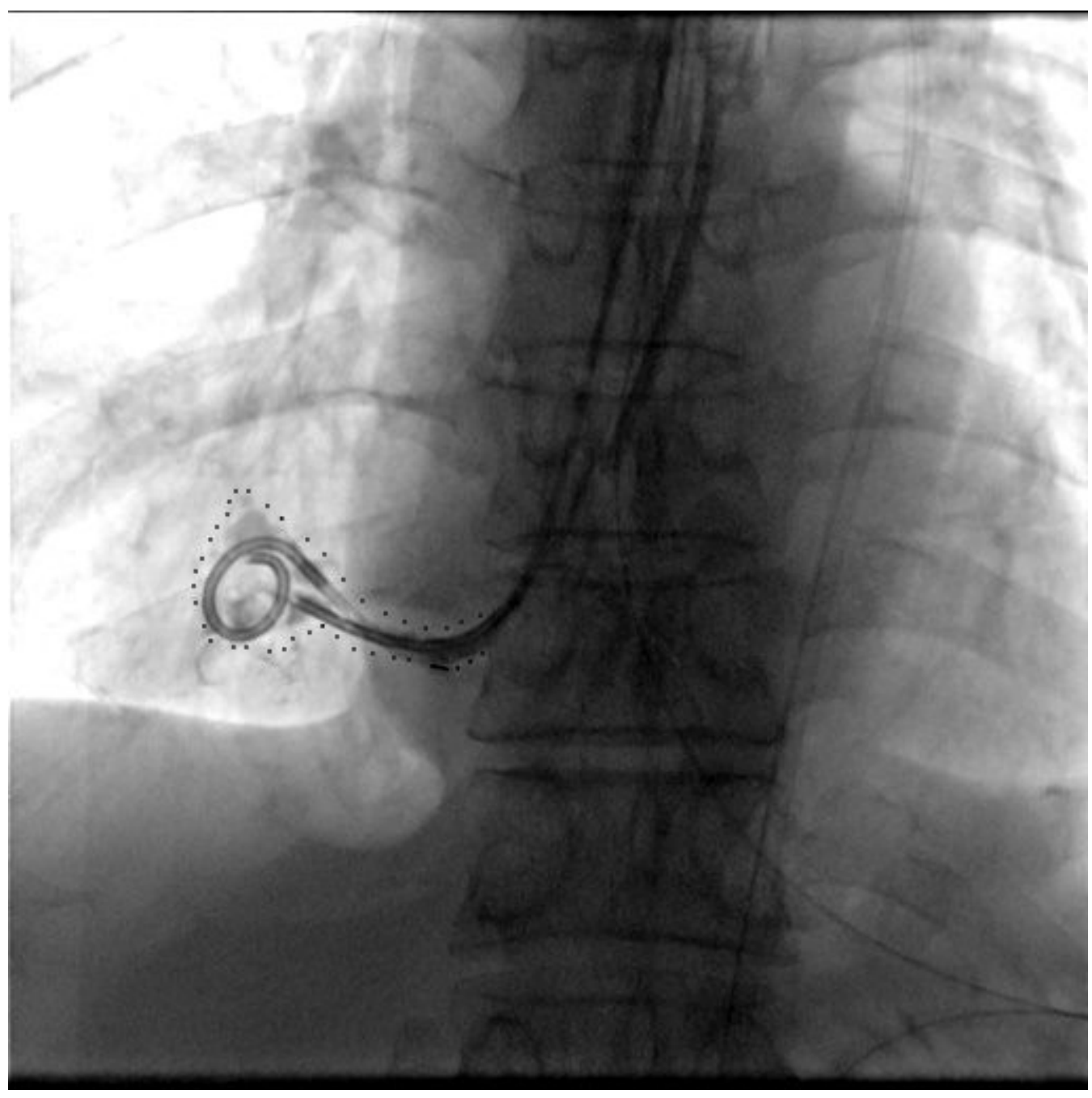

\section{Figure 2}

Radiography shows the abscess drainage tube in position and a decrease in the abscess cavity size on fourth follow-up after the interventional procedure (dots show the outline of the abscess cavity).

\section{Supplementary Files}

This is a list of supplementary files associated with this preprint. Click to download.

- SupplementaryFigureLegend.docx

- supplementaryfigure1.png

- supplementaryfigure2.png

- supplementarytables.xlsx 